\title{
Über den dynamischen Begriff des Nährwertes.
}

\section{Eine Bemerkung zu der Arbeit:von J. König in dieser Zeitschrift'1).}

\author{
Von
}

C. Oppenheimer in München.

[Eingegangen am 12. Juni 1918.]

Es ist mit großer Freude zu begrüßeu, daß auch die praktischen Vertreter der Nahrungsmittelkunde sich im Anschluß an die energetische Betrachtungsweise bemühen, den Begriff des Nährwertes fester zu formen und wissenschaftlich zu vertiefen, wie dies J. König im Anschluß an die Erwägungen getan hat, die Rudolf Höber und ich angestellt haben. Wenn auch Einverständ̦is därüber herrscht, daß uns zur Zeit die Einführung des Begriffes der „maximalen Arbeit" bezw. der freien Energie in die Nährstofflehre kaum Vorteile in praktischer Hinsicht gewährte, so ist es doch auch jetzt schon von großem Werte, wenn die Nahrungsmittelchemiker sich daran gewöhnen, den Kalorienbegriff als einen praktischen Notbehelf anzusehen, und sich über die theoretischen Grundlagen der Auswertung der Energie im Tierkörper klar zu werden.

Unter diesen Umständen ist es aber notwendig, daß wirklich volle Klarheit herrscht, und deswegen gestatte ich mir darauf hinzuweisen, daß die Ausführungen von König an einer wichtigen Stelle mißverständlich ausgelegt werden können. König schreibt unter Beziehung auf den Rubner'schen Begriff der "spezifischdynamischen Wirkung“ der Eiweißkörper: „Ebenso ist der Bruchteil, der von der Verbrennungswärme im Körper als dynamische (freie) und als thermische Energie auftritt, bei den einzelnen Nährstoffen verschieden groß."

Diese Ausdrucksform könnte zu der mißverständlichen Ansicht führen, als wäre in Wirklichkeit bei der Umsetzung selbst ein von der Temperatur abhängiges Verhältnis zwischen der Abgabe thermischer und freier Energie bei den Eiweißkörpern festzustellen. Dies ist aber nicht der Fall, oder wenigstens nicht mit Sicherheit festzustellen. Das Rubner'sche Phänomen liegt vielmehr darin, daf die Eiweißkörper unter allen Um. ständen bei Umsetzung kalorisch gleicher Mengen in den Geweben des Tierkörpers einen größeren Bruchteil von Wärme im Verbältnis zu der disponiblen freien Energie abgeben, als Fette und Kohlenhydrate. Die Beziehungen der Temperatur der Umgebung zu diesem Phänomen liegen nur in der Versehiedenheit, wie diese überschüssige Wärme zum Vorschein kommt. Befindet sich nämlich das Tier in einer Umgebungstemperatur von etwa $30^{\circ}$, also im Zustande der physikalisehen Wärmeregulation, so genügt die ohnehin bei den Zellprozessen abfallende Wärme, um sein Wärmebedürfnis zu decken; und dann wird die beim Umsatz der Proteine entfallende überschüssige Wärme, ohne weiteres als solche .zutage treten und damit eine Mehrabscheidung von Wärme bei dem eiweißgefütterten Tier, gegenüber dem mit Fetten bezw. Kohlenhydraten gefütterten, meßbar sein. Dann ist also das Phänomen der spezifisch.dynamischen Wirkung der Eiweilkörper klar ausgesprochen.

Befindet sich dagegen das Tier in einer kühlen Umgebung, so mul es sein Wärmebedürfnis ,chemisch regulieren", und zwar indem es Prozesse der willkürlichen oder unwillkürlichen Muskelarbeit (Zittern) einleitet, eigens zu dem Zweek, die Wärme-

1) Diese Zeitschrift 1918, 35, 217. 
produktion seiner Gewebe zu erhöhen. Gibt man einem Tier in diesem Zustande Eiweißkörper, so kann die überschüssig gebildete Wärme ersichtlich dazu verwendet werden, um dieses stärkere Wärmebedürfnis zu befriedigen, und dadurch die eben erwähnten Prozesse der chemischen Wärmeregulation entbehrlich machen; dann verschwindet die Mehrahgabe von Wärme entweder ganz oder wird undeutlich. Der Grund, warum also das Rubner'sche Phänomen nur bei höherer Umgebungstemperatur auftritt, ist ein rein accessorischer, nicht im Wesen der Eiweißumsetzung selbst begründet. Die spezifisch-dynamische Wirkung selbst kann man also ungeachtet dieser accessorischen Verschiebungen ihrer Deutlicbkeit dahin kennzeichnen, dalo unter allen Umständen bei Fütterung von Eiweißkörpern Prozesse verstärkt werden, die zur Wärmeabgabe führen, ohne mit den eigentlich energetischen Leistungen des Körpers nach außen hin, also äußerer Muskelarbeit verknüpft zu sein, und damit wäre also gezeigt, daß der dynamische Nährwert der Eiweißkörper, soweit er eben für die Leistung äußerer Muskelarbeit in Betracht kommt, unter allen Umständen um etwa $15 \%$ niedriger ist als der kalorische, durch die Wärmeabgabe charakterisierte Nährwert.

Man kann aber den kalorischen Energiewert auffassen als die Gesamtheit derjenigen Prozesse, die den sogenannten Ruhestoffwechsel ausmachen; dieser umfaßt ebensowobl rein chemische Vorgänge bei der Zellarbeit, wie physikalisch-chemische Arbeitsleistungen, insbesondere osmotische und Sekretionsarbeit, wie endlich auch innere Muskelarbeit des Herzens, der Atemmuskeln u. dergl. In dem Begriff des Ruhestoffwechsels und seiner kalorischen Auswertung summieren sich also eine ganze Reihe von wärmeabgebenden Prozessen gänzlich verschiedener Natur. Einerseits handelt es sich um Wärmeabgaben, die ich als die primäre Wärme bezeichnen möchte, nämlich um Wärmesummen, die bei den zum Teil irreversiblen chemischen Umsetzungen in den Zellen direkt frei werden, andererseits un sekundäre Wärme, die durch Aufhebung ursprünglich geleisteter innerer Arbeit infolge von Reibung entsteht, als Endresultat der genannten Drüsen- und Muskelarbeiten.

Das Wesen der spezifisch-dynamischen Wirkung der Eiweißkörper liegt nun darin, daß sie sich ganz ausschließlich auf die calorisehen Summen dieses Ruhestoffwechsels bezieht. Wie ich in meiner von $\mathrm{K}$ önig zitierten Arbeit in der Biochemischen Zeitschrift ${ }^{1}$ ) näher ausgeführt habe, hat $\mathrm{Zuntz}$ direkt bewiesen, daß für die reine energetische Muskelleistung die Umsetzungen der Eiweißkörper im Muskel denselben dynamischen Wert haben, wie die von Kohlenhydraten und Fetten. Der Muskel scheint nach allem, was wir wissen, die in ihm selbst zur langsamen Oxydation gelangenden Nährstoffe mit demselben dynamischen Wirkungsgrad zu verarbeiten.

Wir müssen also. die Ursachen der spezifisch-dynamischen Wirkung im Ruhestoffwechsel suchen, nach dem oben Gesagten also entweder in den Prozessen der rein chemischen Zellarbeit oder auf dem Gebiete der inneren, erst sekundär in Wärme übergehenden osmotischen oder Muskelarbeiten.

In der Tat lassen sich die Gründe des Rubner'schen Phänomens hier wenigstens im Prinzip auffinden, wie ich ebenfalls in meiner obengenannten Arbeit näber ausgeführt habe.

An dieser Stelle sei nur kurz daran exinnert, daß das $R u b n e r$ 'sche Phänomen eine komplizierte Erscheinung ist, die mindestens drei Wurzeln hat. Erstens wirken

1) Biochem. Zeitschr. 1917, 79, 302 . 
die Eiweißkörper und ihre im Stoffwechsel entstehenden Spaltprodukte, Aminosäuren und Harnstoff, steigernd auf die Tätigkeit der Zelle selbst. Es wird damit der Umsatz der Zelle direkt erhöht, und mit diesem Umsatz naturgemäßo die Wärmeerzeugung. Es ist dies also eine reine Reizwirkung.

Zweitens aber bedingt die Verarbeitung der Eiweißkörper einen erböhten Aufwand an Arbeit gewisser Syeteme, die Zuntz unter den Begriff der Verdauugsarbeit zusammengefaßt hat. Höchstwahrscheinlich handelt es sich in der Hauptsache bei den Eiweibkörpern um eine Erhöhung der Sekretion der Darmdrüsen, die naturgemäß mit einem erhöhten Energieanspruch dieser Systeme und damit erhöhter Wärmeabgabe verbunden ist.

Der dritte und theoretisch interessanteste Grund des $R u b n$ er'schen Phänomens ist, wie auch $R$ ubner selbst mehrfach angedeutet hat, in vorbereitenden Umwandlungsprozessen der Eiweißkörper zu suchen, die nicht im Muskel, sondern an anderen Stellen des Körpers, wohl hauptsächlich in der Leber, verlaufen, und mit einer Verminderung der freien Energie der Eiweißkörper verbunden sind, ohne dab diese Energieabgabe aus den ebenerwähnten räumlichen Gründen der Muskelarbeit zugute kommen kann. Wie ich in meiner mehrfach erwähnten Arbeit auseinandergesetzt habe, ist es als ausgeschlossen zu betrachten, daß Eiweißkörper selbst oder die mit sehr geringem Abfall des Energiepotentials aus ihnon entstandenen hydrolytischen Spaltstücke (Aminosäuren) im Muskel selbst zur Oxydation gelangen. Sie werden zweifellos vorher durch Abspaltung des Stickstoffes chemisch verändert, und nur diese stickstofffreien, bereits etwas oxydierten Bruchstücke gelangen zur definitiven Oxydation in die Muskelmaschine, und zwar kommen sie dorthin bereits mit einem verminderten Vorrat an freier Energie. Diese Stoffe aber werden dann nach Maßgabe der noch in ihnen vorhandenen verfügbaren Energie ebenso ausgenutat wie Fette und Kohlenhydrate, und dies ist der Grund, warum man bei Berechnung der reinen äuBeren Arbeit, also nach Abzug sämtlicher auf den Ruhewert zu beziehenden Energiesummen, in der Tat keinen dynamischen Unterschied zwischen Eiweißkörpern und stickstofffreien Nährstoffen auffinden kann, wie dies Zuntz nachgewiesen hat.

Wir kommen also bei der Erklärung des Rubner'schen Phänomens zu derselben Auffassung, wie sie vor vielen Jahren Chauveau irrtümlich für die Fette angenommen hat. Er glaubte nämlich aus Versuchen den Schluß ziehen zu können, då die Fette überhaupt nicht als solche im Muskel oxydiert werden, sondern vorher in der Leber unter Energieverlust in Zucker übergeführt werden müssen, und schrieb infolgedessen den Fetten eine um etwa $25 \%$ verminderte dynamische Wirkung gegenüber den Kohlenbydraten zu. Diese Ansicht konnte widerlegt werden, aber die grundlegende Idee läßt sich mit besserer Begründung auf die Eiweißkörper übertragen, die in der Tat infolge notwendiger chemischer Vorbearbeitung an anderen Stellen des Körpers nicht mit ihrem vollen kalorischen Wert dynamisch im Muskel zur Wirkung kommen können.

Insofern sind also tatsächlich die Eiweißkörper für energetische Zwecke nur dann ein vollwertiges Material, wenn gleichzeitig ihre überschüssige Kalorienabgabe eine zweckmäßige Verwendung im Wärmehaushalt finden kann, wie dies im allgemeinen bei kühler Umgebung der Fall sein wird. Aber diese mehr zufällige Verwendungsmögliehkeit überschüssig abgegebener Wärmemengen hat ihre Grenze; und wenn wir davon absehen, müssen wir in der Tat feststellen, daß der dynamische Nährwert der 
Eiweißkörper um einen nicht unerheblichen Prozentsatz hinter dem der stickstofffreien Nährsubstanzen zurücktritt, bei denen derartige Verluste durch außerhalb des Muskels verlaufende exothermische Vorgänge entweder nicht vorhanden sind oder sich in sehr viel geringeren, im Stoffwechselversuch nur unsicher zu fassenden zahlenmäßigen Grenzen halten.

\title{
Die Unterseheidung von Molkeneiweiß und Quark.
}

\author{
Von \\ 0. Lüning und W. Tömius.
}

Mitteilung aus der Nahrungsmitteluntersuchungstelle an der Herzoglich Technischen Hochschule Brauschweig.

[Eingegangen am 23. Juni 1918.]

Während vor dem Kriege die in den Molken zurückbleibenden EiweiBstoffe fast ausschließlich durch Verfütterung der Molken verwendet wurden, weil ihre Abscheidung zu kostspielig war, hat der im Laufe des Krieges eintretende große Mangel an eiweißhaltiger Nahrung eine ausgedehnte Gewinnung des Molkeneiweißes veranlaßt. Da die Gestehungskosten des Molkeneiweißes wegen der erforderlichen, verhältnismäßig großen Menge Feuerung höher sind als die des Quarks, sind vielerorts für Molkeneiweiß höhere Höchstpreise festgesetzt als für Quark. Dieser Umstand bringt es mit sich, daß von gewinnsüchtigen Händlern Quark allein oder in Gemischen mit Molkeneiweiß unter der Bezeichnung Molkeneiweiß und natürlich zu dem höheren Preise des Molkeneiweißes verkauft wird. In anderen Gegenden, in denen sich wahrscheinlich das Molkeneiweiß weniger gut als solches verwerten ließ, ist es vorgekommen und kommt es vielleicht noch vor, daß es dem Quark bei Herstellung von Käse untergemischt wurde, ein Verfahren, das nach Ansicht des Kriegsernährungsamtes durch $\S 5$ der Bundesratsverordnung vom 20. Oktober 1916 verboten ist.

Infolgedessen gewinnt die Frage praktische Bedeutung: Wie ist Molkeneiweiß von Quark zu unterscheiden bezw. in Gemisehen beider nachzuweisen?

Bei den Versuchen zur Beantwortung dieser Frage gingen wir anfangs davon aus, daß das Casein einen relativ hohen Phosphorgehalt hat, während das Milchalbumin frei von Phosphor ist. Es zeigte sich jedoch, daß irgendwelche kennzeichnende Unterschiede im Phosphorgehalt der Caseinasche und der Asche des Molkeneiweißes nicht bestanden, da auch diese viel Phosphorsäure enthielt. Vermutlich erklärt sich dér hohe Phosphorgehalt auch des Molkeneiweißes daraus, daß bei seiner Gewinnung Phosphate mit zur Ausscheidung gelangen. Mit der näheren Erforschung der Ursache haben wir uns nicht befaßt, da es uns in erster Linie um die Auffindung einer praktisch brauchbaren Unterscheidung des Molkeneiweißes vom Quark zu tun war.

Die Möglichkeit einer solchen Unterscheidung scheint uns nun in der Löslichkeit des Caseins in Natriumoxalatlösung gegeben, die schon von $\mathrm{Baier}^{\mathbf{1}}$ ) zur Bestimmung des Caseins in Milchschokolade benutzt worden ist.

Casein und somit auch Quark Iösen sich in 1\%-iger Natriumoxalatlösung auf, und aus der Lösung läßt sich das Casein durch Essigsäure wieder zur Abscheidung bringen. Molkeneiweil gibt, mit der gleichen Natriumoxalatlösung behandelt, an diese

1) Diese Zeitschrift $1909,18,13$. 